\title{
D'un simple dessin de Léonard de Vinci aux "formes premières"
}

\section{From a simple drawing of Leonardo da Vinci to "first forms"}

\author{
Jean-Pierre Crettez ${ }^{1}$ \\ ${ }^{1}$ Chercheur émérite à Telecom Paris
}

RÉSUMÉ. L'analyse géométrique d'un dessin représentant le profil d'une tête d'homme, nous laisse entrevoir comment Léonard établissait sa démarche constructive des profils à partir d'une forme première. L'analyse géométrique de trois autres profils de Léonard conforte cette approche. C'est avec cette même approche que Léonard a réalisé ses cinq portraits féminins.

ABSTRACT. The geometric analysis of a drawing representing the profile of a man's head gives us a glimpse of how Leonardo established his constructive approach to profiles from a first form. The geometric analysis of three other Leonardo profiles confirms this approach. It is with this same approach that Leonardo produced his five female portraits.

MOTS-CLÉS. commensurabilité, divine proportion, forme elliptique, forme première, géométrie interne, géométrie secrète, maillage, nombre d'or, poncif, portrait, profil, stylisation des formes.

KEYWORDS. commensurability, divine proportion, elliptical form, first form, internal geometry, secret geometry, mesh, golden ratio, pouncing pattern, portrait, profile, stylization of forms.

\section{Introduction}

Les conseils, préceptes et directives techniques qui émanent des nombreux écrits et dessins que nous a laissés Léonard, nous conduisent à considérer la peinture comme une science dont le but est la re-création du monde visible. "Cette idée que l'art est une création amène Léonard à insister sur le fait que le peintre doit être un esprit universel qui ne néglige aucun aspect de la nature. Il doit être un savant, c'est-à-dire qu'il doit comprendre les mécanismes intérieurs de ce qu'il peint, presque comme s'il en était le créateur."

"Sachant, ô peintre que pour être excellent, tu dois avoir une aptitude universelle à représenter tous les aspects des formes produites par la nature..."

Mais pour Léonard, les formes créées par la nature ne sont pas aléatoires, elles sont dues à la Nécessité. "Nécessité est maitresse et tutrice de la Nature..."

"Necessity dictates the form always perfectly fits function in nature, with no insufficiency or redundancy; it compels every force to expend itself in the most direct way available to it; it prescribes that the simplest design to achieve a given end will be followed; and it must be respected by any human contriver of artificial things... The universal architecture of Necessity is geometry."

Ainsi, le peintre doit être un savant, il doit comprendre les lois internes de la Nature et les principes de la Nécessité. Léonard conseille à ses élèves: "Celui qui apprend la peinture doit

\footnotetext{
${ }^{1}$ Clark K.,[3], p.158

${ }^{2}$ Léonard de Vinci, Ms. 2038 Bib. nat. 4 r.

${ }^{3}$ Léonard de Vinci, Forster III, 43v.

${ }^{4}$ Kemp M.,[7], p. 83
} 
posséder des connaissances mathématiques.". Bien plus, il doit savoir établir la perspective: "la perspective est la première chose qu'un jeune peintre doit apprendre pour savoir mettre chaque chose à sa place, et pour lui donner la juste mesure qu'elle doit avoir dans le lieu où elle se trouve ${ }^{6 "}$. Et il doit aussi savoir appliquer la théorie des proportions: "la peinture repose sur la proportion des parties considérées les unes par rapport aux autres, et au tout qu'elles composent ${ }^{7}$ ". Enfin, Léonard, influencé par Alberti, “... suggère pour la composition harmonieuse des formes proportionnelles entre elles, un système d'équivalence avec certains intervalles musicaux 8 ".

Malgré toutes ces recommandations à caractère géométrique, Léonard ne nous a pas transmis d'exemples de construction géométrique des formes ni de leur évolution, ni comment ces formes pouvaient être idéalisées. Nous pouvons supposer que Léonard réservait ces exemples pour son traité: le "de pictura et movimenti humani" aujourd'hui disparu, ou encore pour un projet qui n'a jamais vu le jour: le de ludo geometrico.

Pour tenter de retrouver certains indices concernant la démarche constructive de Léonard, nous avons analysé un simple dessin représentant le profil d'une tête d'homme. Ce dessin est transcrit dans un espace libre d'un des feuillets de son Manuscrit A. Par comparaison avec notre étude et pour corroborer nos résultats, nous avons analysé une autre tête d'homme vue de profil, dessinée dans le Codex Atlanticus. Enfin, nous avons complété notre étude avec l'analyse de deux profils féminins célèbres: celui de la Belle Princesse (Bibliothèque Narodowa à Varsovie) et le portrait d'Isabelle d'Este (Louvre).

\section{Le profil du Manuscrit A}

Le folio ${ }^{9} 2 \mathrm{v}$ du Manuscrit $A$ (Figure 1 à gauche) est consacré à la description du principe de la balance romaine. Il présente, sur la droite, un dessin sans commentaire représentant le contour d'une tête d'homme vue de profil. Une ligne courbe (figure 2) entoure le devant de la figure. Tandis qu'à droite, sur le folio $3 r$, Léonard a écrit un texte relatif à la science perspective. Cette diversité des thèmes abordés que l'on retrouve dans tous ses carnets, réunissant plus de 6000 pages de notes, de croquis, de réflexions et d'études scientifiques, reflète l'universalité de ses domaines d'intérêt.

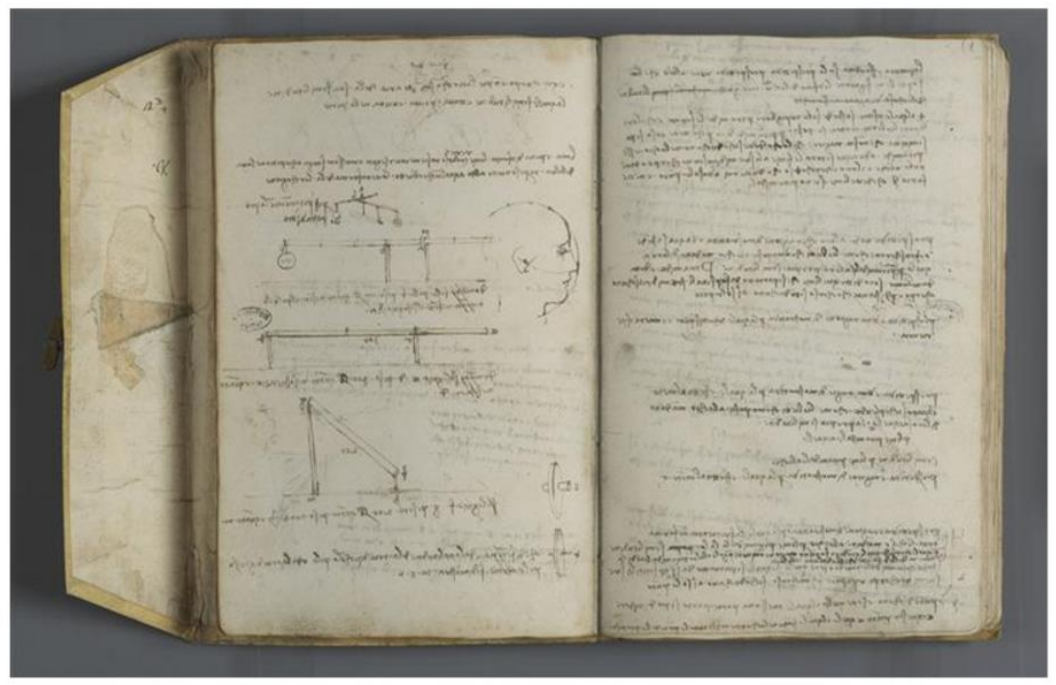

Figure 1. Folio 2v et $3 r$ du Manuscrit A de L. de Vinci, Bibliothèque de l'Institut de France

\footnotetext{
${ }^{5}$ Léonard de Vinci[10], chapitre II

${ }^{6}$ Léonard de Vinci[10], chapitre I

${ }^{7}$ Léonard de Vinci[10], chapitre XLIX

${ }^{8}$ Clark K.,[3], p.159,

${ }^{9}$ Léonard de Vinci[9|], Folio $2 \mathrm{v}$
} 
Le Manuscrit A, n'est pas le premier des 14 Manuscrits écrits par Léonard. Il date des années 1490-1492. Cependant, puisque le dessin a été inséré dans une partie laissée vide du manuscrit, il est possible de supposer que ce dessin soit postérieur à l'écriture de ce manuscrit et même, puisqu'il est sans commentaire, qu'il ne soit pas de Léonard.

Depuis 1482, Léonard se trouve à Milan à la cour du duc Ludovic Sforza. Ingénieur-architecte militaire, inventeur, joueur de lyre, costumier, arrangeur de feste, sculpteur, Léonard est aussi un peintre reconnu: il a réalisé L'Annonciation (1473-1475), La Madone à l'oillet (1473), le Portrait de Ginevra de'Benci (1476-1478).

Dans ses portraits, il cherche à montrer le tempérament du modèle, et en particulier sa vie intérieure. En effet, Léonard donne à ses élèves le conseil suivant: "Tu ne feras jamais les têtes droites sur les épaules, mais tournées de côté, à droite ou à gauche, même si elles regardent en haut ou en bas, ou tout droit, parce qu'il est nécessaire de faire en sorte que leurs mouvements aient l'air d'être vivants et non figés ${ }^{10}$." Par contre, à cette époque, les profils sont principalement réservés à la représentation des princes ou des hommes d'état, figés dans une attitude dépourvue de sentiments, comme sur les médailles antiques. C'est peut-être la raison pour laquelle, Léonard, nous a laissé peu de dessins ou de tableaux représentant des têtes vues de profil.

\section{Description de la tête vue de profil}

Sur ce profil, on aperçoit plusieurs points qui ressemblent à des petits amas de poudre, (spolvero) déposés lors de l'application d'un poncif (figure 3). Parmi ces points, certains correspondent au contour du crâne. Un point particulier, situé au creux de l'oreille, peut être associé au centre $\mathbf{O}$ du profil. Un autre point indique le bas de la nuque, et plus bas, un point esquisse la naissance du cou. Visiblement, ce dessin a été transcrit à partir d'un poncif c'est-à-dire d'un patron percé de petits trous. La méthode du poncif était courante dans l'atelier de Léonard. Plusieurs réflectographies infrarouges de ses œuvres montrent des contours parsemés d'une série de points de spolvero ${ }^{11}$. À partir de ces points, le transcripteur a dessiné le profil de la tête, et précisé le visage. Mais, il a aussi reporté cette courbe régulière qui frôle le menton, le nez, le front, et le haut du crâne. Puis il a tracé un arc de cercle allant de l'oreille à la pointe du nez, et un embryon de courbe esquissant l'arrière du crâne, et un autre suggère la naissance du cou.

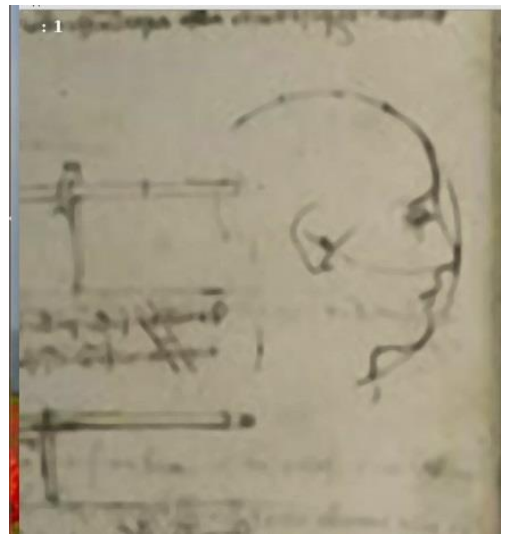

Figure 2. Une courbe régulière entoure le profil de la tête.

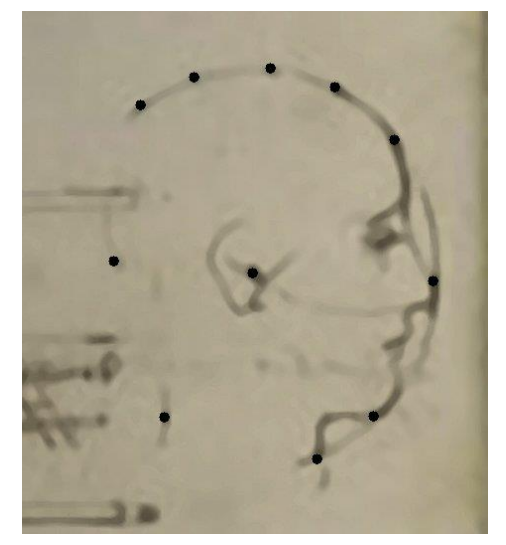

Figure 3. Le profil de la tête et les points du poncif.

Comment était constitué ce patron transformé en poncif? Pourquoi comportait-t-il cette courbe régulière qui entoure le profil? Quelles sont les propriétés de cette courbe? Comment a-t-elle été construite? Pourquoi cette dernière a-t-elle été retracée dans ce dessin ?

\footnotetext{
${ }^{10}$ Léonard de Vinci, Le traité de peinture, note 354, p. 149. Éditions Jean de Bonnot, Paris 1982.

${ }^{11}$ Mottin B.,[8], Léonard de Vinci et l'art du dessin: une approche de laboratoire. Léonard de Vinci, Éditions Hazan 2019.
} 


\section{Forme elliptique de la courbe}

L'auteur du poncif semble avoir effectué son dessin à l'aide de la géométrie. En effet, cette courbe régulière (figure 4), trop étirée pour être un arc de cercle, correspond précisément à un arc d'ellipse. L'ellipse correspondante, centrée au point $\mathbf{O}$, est orientée verticalement. Elle a pour demi-grand axe $\mathbf{a}=\mathbf{O A}$, et pour demi-petit axe $\mathbf{b}=\mathbf{O B}$.

La distance focale de l'ellipse $\mathbf{f}=\mathbf{O F}$ se détermine (figure 5) en traçant un arc de cercle de centre $\mathbf{B}$ et de rayon $\mathbf{B M}=\mathbf{a}$. Cet arc de cercle coupe la droite $\mathbf{O A}$ au point $\mathbf{F}$, définissant ainsi, la distance focale $\mathbf{f}=\mathbf{O F}$. Par suite, les côtés du triangle rectangle $\mathbf{O B F}$ vérifient la relation de Pythagore, propre aux ellipses : $\mathbf{a}^{\mathbf{2}}=\mathbf{b}^{\mathbf{2}}+\mathbf{f}^{\mathbf{2}}$. L'autre foyer $\mathbf{F}^{\prime}$, tel que $\mathbf{O F} \mathbf{F}^{\prime}=\mathbf{f}$, s'obtient par symétrie par rapport au point $\mathbf{O}$.

On remarque (figure 6) que le rectangle OBM'F est un rectangle double-carré, et par suite que la distance focale $\mathbf{f}$ est égale à la moitié du demi-petit axe $\mathbf{:} \mathbf{f}=\mathbf{b} / \mathbf{2}$. L'auteur du poncif a donc choisi une ellipse particulière pour effectuer son dessin. Elle est telle que $\mathbf{a}^{\mathbf{2}}=\mathbf{4} \mathbf{f}^{\mathbf{2}}+\mathbf{f}^{\mathbf{2}}=\mathbf{5} \mathbf{f}^{\mathbf{2}}$. Son excentricité est $\boldsymbol{\varepsilon}=\mathbf{f} / \mathbf{a}=\mathbf{1} / \sqrt{ } \mathbf{5}$. C'est, suivant notre classification ${ }^{12}$, une ellipse arrondie de type $\mathbf{5}$. (Cent trente ans plus tard, Georges de La Tour modélisera le bord de la toque de $\operatorname{Joseph}^{13}$ dans L'Adoration des bergers, par une ellipse de même excentricité). Cependant, même si l'auteur a choisi ici, une ellipse particulière, la méthode exposée ci-dessous reste générale.

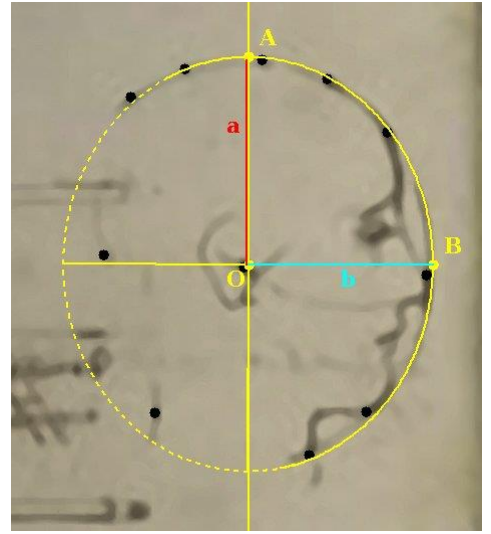

Figure 4. Le tracé de l'ellipse

\section{Maillage carré}

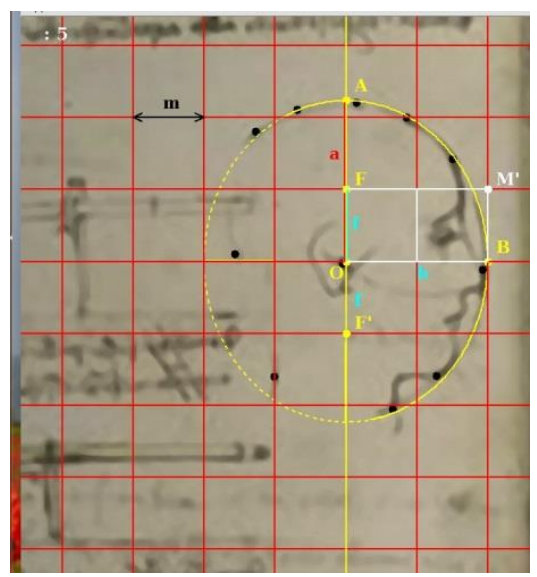

Figure 6. Le maillage carré.

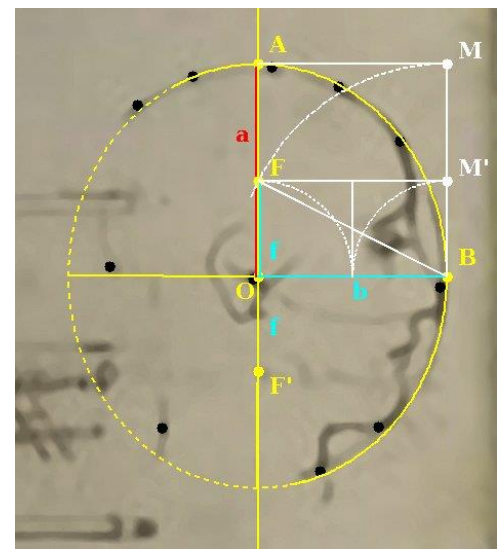

Figure 5. Les 3 paramètres $a, b$, $f$ de l'ellipse.

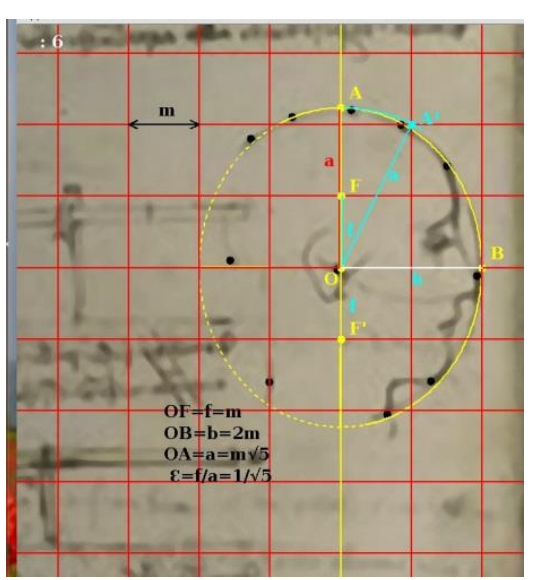

Figure 7. Valeur des paramètres de l'ellipse.

\footnotetext{
${ }^{12}$ Crettez J-P.,[4], §. 2.4.4.

${ }^{13}$ Ibidem, §. 2.4 .5
} 
Ce rectangle double carré, appelé figure de diapason ${ }^{14}$, peut être considéré comme un maillage carré élémentaire que l'on peut étendre à tout le dessin (figure 6). Nous avons pris pour unité de mesure de ce dessin, la largeur ${ }^{15} \mathbf{m}$ de la maille.

\section{Valeur des paramètres de l'ellipse}

L'auteur a probablement construit l'ellipse sur ce maillage. La distance focale est égale à la largeur de la maille $: \mathbf{f}=\mathbf{m}$. Le demi-petit axe $\mathbf{b}$ vaut deux largeurs de maille $\mathbf{:} \mathbf{b}=\mathbf{2} \mathbf{m}$. Et le demigrand axe a est égal (figure 7) à la diagonale de deux mailles superposées : $\mathbf{a}=\mathbf{m} \sqrt{\mathbf{5}}$.

\section{La divine proportion}

Cette ellipse particulière a probablement été choisie parce qu'elle est associée au nombre d'or $\boldsymbol{\Phi}=$ 1,618. En effet, sur l'axe vertical médian, le foyer $\mathbf{F}$ divise (figue 8), le segment $\mathbf{A} \mathbf{F}^{\prime}$ en deux segments: FA et $\mathbf{F F}^{\prime}$ selon la divine proportion ${ }^{16}$.

Nous avons : FA/FF' $=\mathbf{F F}^{\prime} / \mathbf{F}^{\prime} \mathbf{A}=\mathbf{1} / \mathbf{\Phi}$, soit $(\sqrt{5}-1) / 2=2 /(\sqrt{ } \mathbf{5}+1)=\mathbf{1} / \mathbf{\Phi}$.

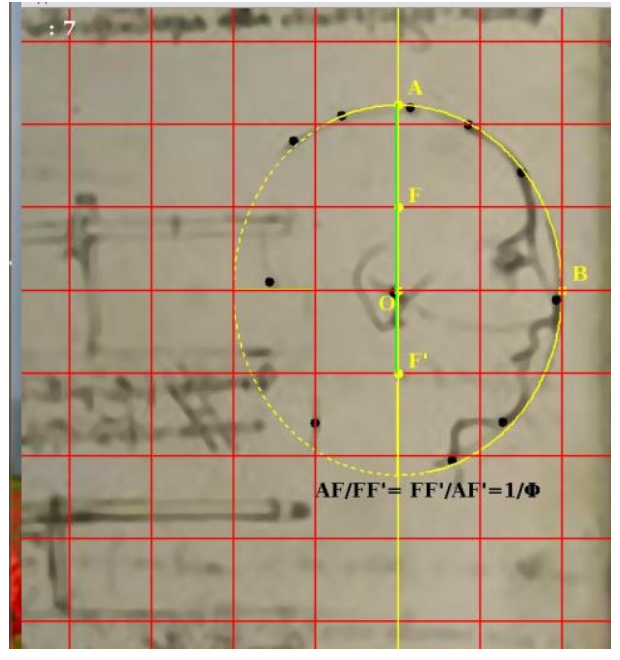

Figure 8. La divine proportion du segment $A F^{\prime}$

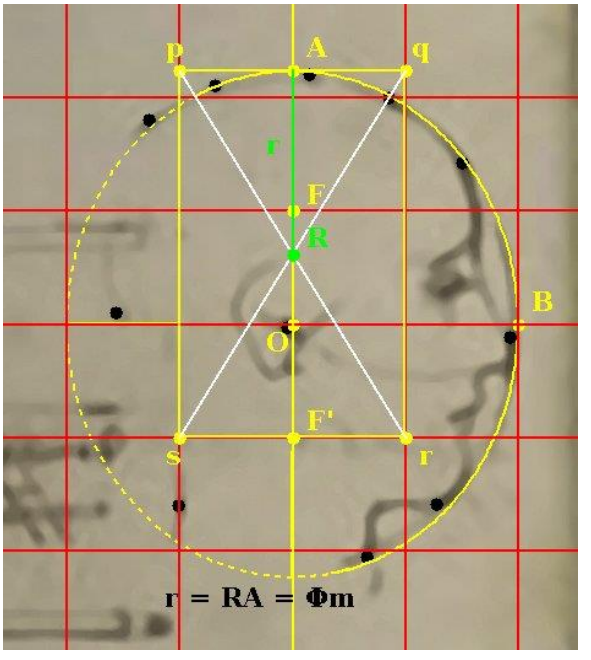

Figure 9. Le rectangle d'or.

\section{Rectangle d'or.}

La distance $\mathbf{A F}=\mathbf{f}+\mathbf{a}=\mathbf{m}+\mathbf{m} \sqrt{\mathbf{5}}=\mathbf{m}(1+\sqrt{\mathbf{5}})=\mathbf{2 \Phi \mathbf { m }}$. Elle vaut deux fois le nombre d'or multiplié par l'unité de mesure $\mathbf{m}$. Par suite, (figure 9), le rectangle pqrs de hauteur $\mathbf{2 \Phi m}$ et de largeur $\mathbf{2 m}$, placé symétriquement par rapport à l'axe vertical médian, a pour format $\mathbf{\Phi}$. C'est un rectangle d'or. Il a pour centre le point $\mathbf{R}$, et le segment $\mathbf{R A}=\mathbf{A F}$ '/2 a pour valeur $\mathbf{\Phi m}$.

\section{La courbe du crâne et de la nuque}

Dans le dessin du Manuscrit A de Léonard (figure 3), on aperçoit un début de courbe esquissant l'arrière du crâne, et un autre suggérant la naissance du cou. Ces deux portions de courbe

\footnotetext{
${ }^{14}$ Bouleau Ch.,[2], La géométrie secrète des peintres. Éditions du Seuil (1963).

${ }^{15}$ Connaissant les dimensions du folio, nous pouvons estimer l'unité de mesure: $\mathbf{m}=0,75 \mathrm{~cm}$.

${ }^{16}$ La proportion divine non donné par le moine franciscain et mathématicien Luca Pacioli, pour exprimer le partage d'un segment en extrême et moyenne raison, selon la définition proposée trois siècles avant notre ère par le mathématicien Euclide. La divine proportion partage un segment donné en deux autres segments, un grand et un petit, de telle façon que le rapport du plus petit au plus grand est égal au rapport du plus grand à la somme des deux. Et ce rapport est égal à 1/Ф.
} 
correspondent à un contour bien structuré, tracé sur le patron, et constitué de deux arcs de cercle (figure 10).

Un premier arc de cercle centré au point $\mathbf{R}$, et de rayon $\mathbf{r}=\mathbf{R} \mathbf{A}=\mathbf{\Phi} \mathbf{m}$, qui prolonge l'arc d'ellipse au point $\mathbf{A}$, fait le tour de l'arrière du crâne, en passant par un point du poncif et va jusqu'au point d'inflexion $\mathbf{I}$.

Et un deuxième arc de cercle situé dans le prolongement, centré en $\mathbf{K}$ et de rayon $\mathbf{r}{ }^{\prime}=\mathbf{K I}=$ $(\boldsymbol{\Phi}+\mathbf{1}) \mathbf{m}=\boldsymbol{\Phi}^{\mathbf{2}} \mathbf{m}$, montre la nuque et la naissance du cou, en passant par un autre point du poncif.

Le segment KR est la diagonale du rectangle $\mathbf{R}$ 'RHK qui a pour côtés: $\mathbf{K H}=(\mathbf{2}+\boldsymbol{\Phi}) \mathbf{m}$, et $\mathbf{R H}=$ $(\mathbf{1} / \boldsymbol{\Phi}+\boldsymbol{\Phi}) \mathbf{m}$. Comme KH/RH $=(\mathbf{2}+\boldsymbol{\Phi}) /\left(\left(\mathbf{1}+\boldsymbol{\Phi}^{\mathbf{2}}\right) / \boldsymbol{\Phi}\right)=\boldsymbol{\Phi}$, le rectangle $\mathbf{R}$ 'RHK est un rectangle d'or. Le point d'inflexion I divise le segment $\mathbf{K R}$ en deux segments: KI et IR selon la divine proportion. Nous avons ainsi: IR/IK $=\mathbf{I K} / \mathrm{KR}=\mathbf{r} / \mathbf{r}^{\prime}=\boldsymbol{\Phi} /(\boldsymbol{\Phi}+\mathbf{1})=\boldsymbol{\Phi} / \boldsymbol{\Phi}^{2}=\mathbf{1} / \boldsymbol{\Phi}$.

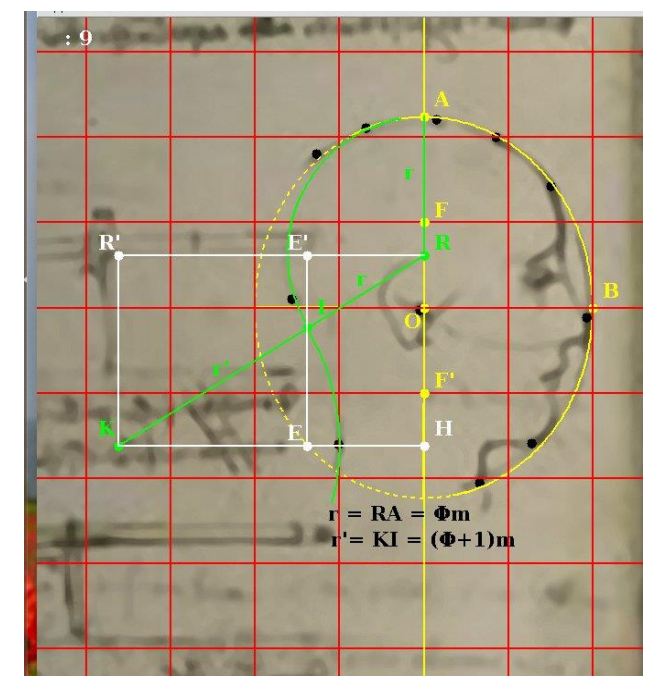

Figure 10. La courbe du crâne et de la nuque

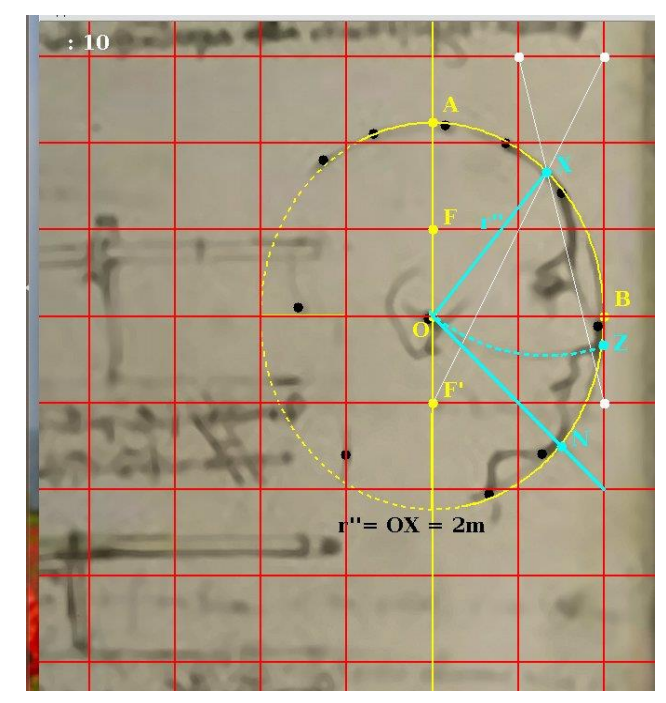

Figure 11. Les trois points d'appui :X, Z, N

Ce contour (tracé en vert, figure 10) du crâne et de la nuque qui nous semble naturel, est géométriquement parfait: on peut observer au point $\mathbf{A}$, la continuité du premier arc avec l'arc de l'ellipse du visage (même tangente), et aussi au point d'inflexion $\mathbf{I}$, la continuité de ce premier arc avec le second (même tangente).

\section{Le devant du profil}

À droite, la forme elliptique partant du haut du crâne, suit la courbure du front, passe par la pointe du nez et le menton.

Le dessin (figure 3) présente un arc de cercle qui part du creux de l'oreille et passe juste sous le nez. Cet arc de cercle est centré à la naissance du front, au point $\mathbf{X}$, intersection de l'ellipse avec des droites tracées sur le maillage (figure 11) et se termine au bout du nez $\mathbf{Z}$. Le point $\mathbf{X}$ a pour coordonnées $(4 \mathbf{m} / 3,5 \mathbf{m} / 3)$ et l'arc de cercle a pour rayon $\mathbf{r}^{\prime \prime}=2 \mathbf{m}$.

L'extrémité $\mathbf{N}$ du menton, (figure 11) correspond à l'intersection de l'ellipse et de la diagonale tracée sur le maillage, et issue de $\mathbf{O}$.

Ainsi, pour tracer le devant du profil, l'auteur n'a défini que trois points d'appui, qui sont liés à l'ellipse et au maillage et respectent donc les proportions. En s'appuyant sur ces trois points, l'auteur a la possibilité de dessiner le devant du profil, en personnalisant le dessin. C'est une manière d'adapter un modèle standard à la diversité des visages. 
Car, l'auteur, à la recherche d'un processus de formation standard, se heurte inévitablement, à l'infinie diversité de la nature humaine. «Si la nature avait un canon fixe pour les proportions des diverses parties, les traits de tous les hommes se ressembleraient tellement qu'il serait impossible de les distinguer entre eux; mais elle a diversifié les cinq parties du visage, en sorte que tout en ayant établi un canon presqu'universel quant à leur dimension, elle ne l'a pas observé dans chaque cas particulier avec assez de rigueur qu'on ne puisse aisément différencier l'un de l'autre. ${ }^{17}{ }$ '

\section{Discussion}

Nous venons de voir que l'auteur, pour établir ce profil, a choisi une ellipse particulière associée au nombre d'or. Puis, à partir de celle-ci, il a tracé le magnifique contour du crâne et de la nuque (figure 10) en utilisant les propriétés de la divine proportion. Or, nous ne trouvons aucune trace de la divine proportion dans les manuscrits de Léonard, ni dans son Traité de la peinture, ni dans ses travaux mathématiques. Alors quel est l'auteur de ce poncif?

En 1496, à la cour du duc Ludovic Sforza à Milan, Léonard a fait la rencontre du mathématicien Luca Pacioli. C'est le moment où ce dernier rédige son traité De Divina Proportione (1496), pour lequel Léonard va dessiner les 5 polyèdres réguliers, dont l'icosaèdre et le dodécaèdre qui contiennent la divine proportion. Cette dernière ne lui était donc pas étrangère.

Mais pour le moine franciscain, Frère Luca Pacioli, la divine proportion, base de la construction du dodécaèdre, ne pouvait qu'être identifiée à Dieu. En effet, les caractéristiques de la divine proportion concordent avec les attributs divins ${ }^{18}$. Par suite, si l'homme a été créé à l'image de Dieu, les différentes parties du profil de sa tête, doivent avoir été conçues en accord avec la divine proportion.

Kenneth Clark $^{19}$ arrive à des conclusions semblables en faisant référence à Protagoras : «Si l'homme est la mesure de toute chose, l'homme physiquement parfait devait être la mesure de toute beauté, et ses proportions devaient pouvoir, d'une façon ou d'une autre, se réduire en expressions mathématiques et rejoindre ces abstractions parfaites que sont le carré, le cercle et le nombre d'or.»

On peut donc supposer que le poncif étudié ci-dessus, soit le fruit d'une étroite collaboration entre Léonard et Luca Pacioli : le premier pour la recherche d'un processus universel de formation, le second pour son savoir et sa rigueur mathématique.

Néanmoins, si l'on fait abstraction du tracé particulier de la courbure du crâne et de la nuque, la genèse du dessin, obtenue à partir d'une ellipse, est conforme à la démarche créatrice de Léonard. Daniel Arasse ${ }^{20}$ explicite cette démarche de Léonard : «Son approche du réel est morphologique, intéressée à la genèse des formes, concernée par l'analogie des lois fondées sur le principe de proportions géométriques et l'irréductible et infinie diversité des aspects des choses.»

\section{Définition de la forme première}

Ce dessin transcrit dans le Manuscrit $A$ ne comporte aucune explication, mais il possède cette courbe ayant la forme d'un arc d'ellipse, dont la fonction n'est pas d'entourer le profil du visage, mais de participer à sa construction. L'ellipse qui contient cet arc constitue ce que nous appelons la forme première.

\footnotetext{
${ }^{17}$ Léonard de Vinci, Codex Atlanticus 119 v. a.

${ }^{18}$ Luca Pacioli,: "Comme Dieu elle est unique. Comme la Sainte Trinité est une substance en trois personnes, elle est une seule proportion en trois termes. Comme Dieu elle est toujours semblable à elle-même..."

${ }^{19}$ Clark K.,[3], p. 165

${ }^{20}$ Arasse D.,[1], p.110 
Plus généralement, pour réaliser un portrait, Léonard, face au modèle, effectuait d'abord un dessin préparatoire en traçant avec sa main gauche plusieurs esquisses du contour de la tête. En suite, le maître cherchait à représenter la superposition de toutes ces lignes, par une courbe géométrique simple à partir de laquelle il pouvait appliquer les lois internes de la Nature et engendrer ainsi la forme recherchée. Daniel Arasse ${ }^{21}$ précise: « un aspect essentiel de l'art de Léonard tient à ce qu'il veut, en accord avec ce qu'il perçoit du monde, y faire sentir "la formation sous la forme"». Mais, pour Léonard, la forme géométrique simple qui s'approche au plus près de l'esquisse est une ellipse. Elle constitue la forme première.

La forme première possède plusieurs propriétés. Elle suggère au spectateur l'aspect global de la tête. Son centre précise sa position, le grand axe indique son inclinaison, (la tête est droite ou penchée) et l'excentricité indique si la tête est plutôt arrondie ou allongée.

Comme nous l'avons montré, la géométrie interne qui participe à la formation du profil ou du portrait, se détermine précisément à partir de cette forme première et de ses paramètres. Les éléments étant issus de la même forme, sont donc proportionnels entre eux.

Enfin, la pureté géométrique de la forme première contribue partiellement à la stylisation du portrait.

\section{Démarche du peintre.}

Ce dessin, nous a indiqué la démarche suivie par l'auteur du poncif, pour effectuer le tracé du profil. Il a d'abord tracé la forme première. Il a emprunté une partie de cette forme première, pour tracer le contour du front et le haut de la tête; ensuite, à partir des paramètres de cette courbe, il a construit, grâce à la géométrie interne, le contour de la nuque et à la naissance du cou ; puis il a continué le contour en le faisant passer par les points d'appui situés sur la forme première et déterminés eux aussi par la géométrie interne, comme l'extrémité du menton, la pointe du nez, le milieu du front.

Léonard nous a-t-il laissé d'autres exemples de profil ?

\section{La tête vue de profil du Codex Atlanticus.}

Le Codex Atlanticus ainsi nommé pour son format grand atlas $(64 \times 43 \mathrm{~cm})$, est un recueil de dessins et de notes de Léonard de Vinci, il est conservé à la bibliothèque Ambrosienne de Milan.

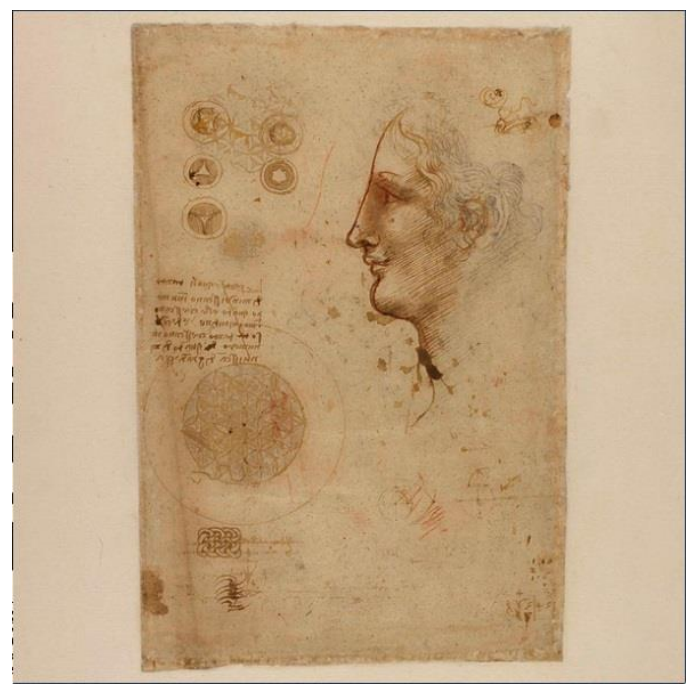

Figure 12. Le feuillet 239 recto du Codex Atlanticus

\footnotetext{
${ }^{21}$ ibidem[1], p. 18
} 
Les dessins de Léonard ont été collés et rassemblés par le sculpteur Pompeo Leoni, en 12 volumes, contenant plus de 1119 feuillets. La rédaction des notes et des dessins s'étend de 1478 à 1519.

Sur le feuillet 239 recto, Léonard a dessiné, à la droite de plusieurs figures géométriques, le profil d'une tête d'homme (figure 12). D'après les spécialistes, le feuillet daterait de 1517, l'année de la mort de Pacioli. Le profil, avec ses ombres et ses lumières est plus achevé que celui du profil du Manuscrit A. L'homme, tourné vers la gauche, regarde droit devant lui, il possède une belle chevelure. L'arrière de sa tête n'est pas représenté. Le profil est visiblement différent de celui du Manuscrit A.

Il n'y a pas de courbe tracée devant lui. Néanmoins, connaissant la démarche constructive de Léonard, cherchant la "formation sous la forme". Nous pouvons supposer qu'il a été inspiré par la méthode de construction du profil du Manuscrit A.

\section{La forme première}

Le profil (figure 13) plus haut que large, la courbure du front et le haut du crâne suggèrent une forme première, constituée d'une ellipse d'axe vertical, centrée au creux de l'oreille. L'ellipse suit l'arrondi du front, frôle la pointe du nez et l'extrémité du menton. L'ellipse est semblable à la forme première du profil du Manuscrit A. Plus précisément, elle possède (figure 14) les mêmes paramètres $(\mathbf{b}=\mathbf{2 f})$ et la même excentricité $\boldsymbol{\varepsilon}=\mathbf{f} / \mathbf{a}=\mathbf{1} / \sqrt{\mathbf{5}}$ que celle de la figure 5 .

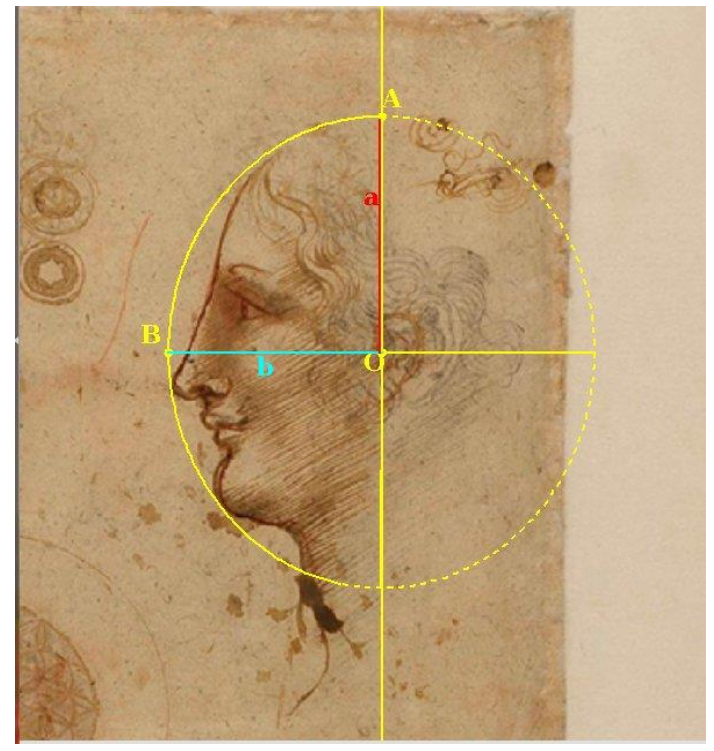

Figure 13. La forme première.

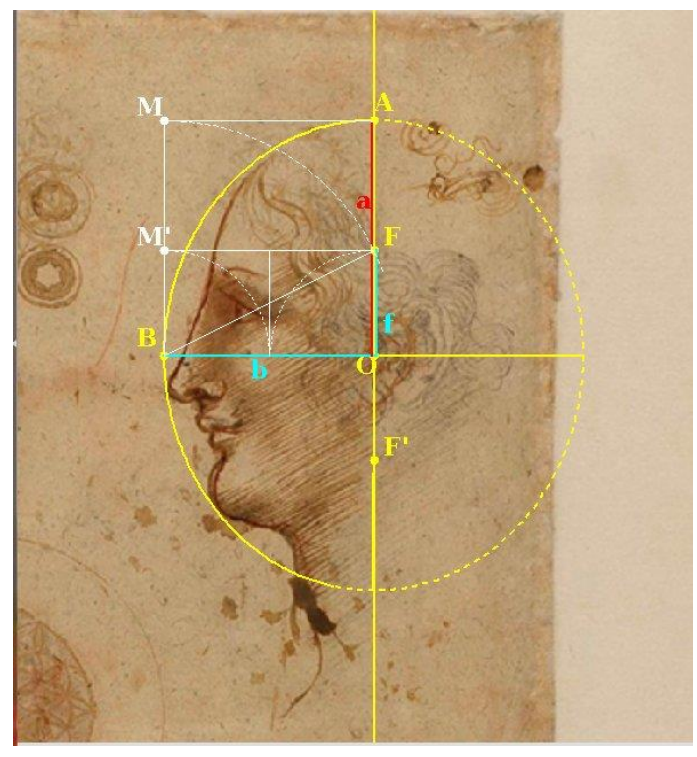

Figure 14. Les paramètres de l'ellipse

Les deux formes premières ne sont pas égales, mais elles sont proportionnelles puisque à cause du format atlante du feuillet, la dimension du profil du Codex Atlanticus est plus grande que celle du Manuscrit A. 


\section{Dessin du profil}

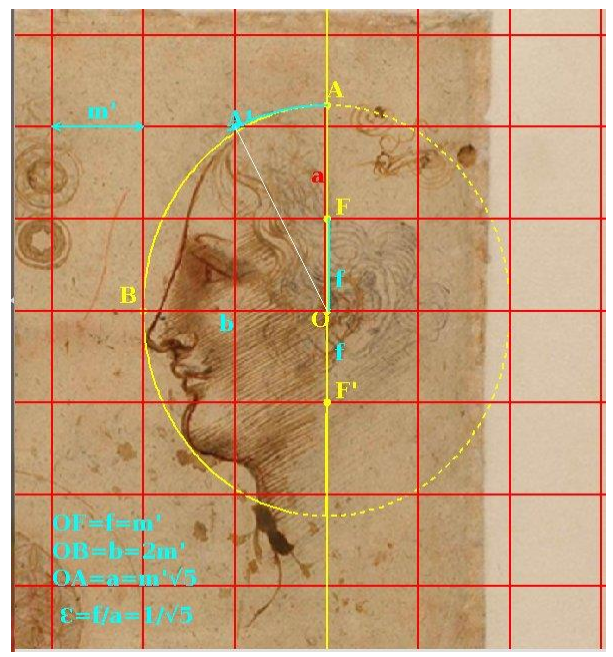

Figure 15. Le maillage carré.

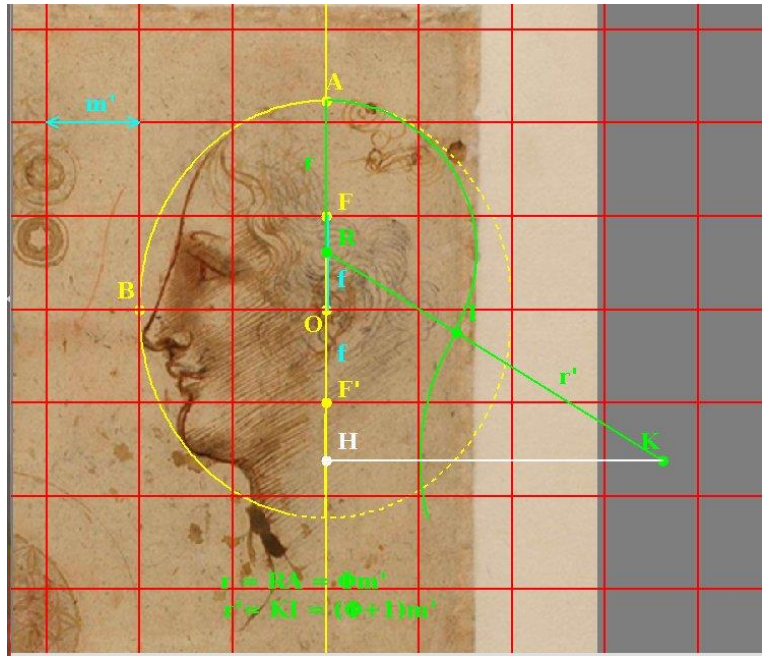

Figure 16. La courbe hypothétique du crâne et de la nuque

Pour réaliser son dessin, Léonard n'a donc pas pu utiliser le poncif créé en collaboration avec Pacioli, il a reconstruit un autre poncif sur un autre patron. Sur ce patron, il a commencé par dresser un maillage carré (figure 15), en prenant pour unité de mesure $\mathbf{m}^{\prime 22}$ la largeur de la maille, il a déterminé les paramètres de l'ellipse : $\mathbf{f}=\mathbf{m}^{\prime}, \mathbf{b}=\mathbf{2} \mathbf{m}^{\prime}$ et $\mathbf{a}=\sqrt{\mathbf{5}} \mathbf{m}$ ' (la diagonale de deux mailles), puis il a tracé l'ellipse constituant la forme première. À partir de celle-ci, il a suivi la méthode exposée ci-dessus.

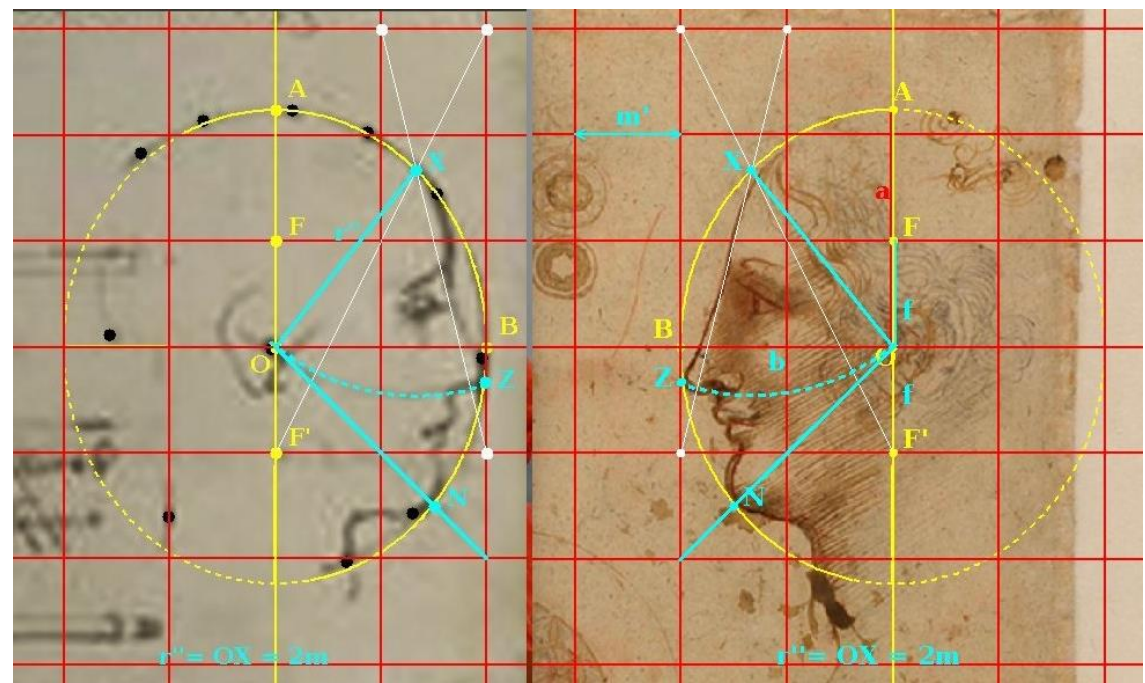

Figure 17. La position des trois points de contact: $X, Z, N$.

En adaptant à la taille et à l'orientation gauche-droite, l'algorithme qui nous a permis d'analyser le profil du Manuscrit A, nous pouvons l'appliquer au profil du Codex Atlanticus, et comparer les deux profils.

Léonard aurait pu tracer la courbe du crâne et de la nuque (figure 16) qui continue la forme première au point A. Mais il n'existe aucune trace de cette courbe sur le dessin. Léonard ne s'est pas intéressé au crâne et à la nuque , mais seulement au-devant du profil.

\footnotetext{
${ }^{22}$ En se basant sur les dimensions du feuillet, nous pouvons estimer l'unité de mesure: $\mathrm{m}^{\prime}=3,96 \mathrm{~cm} .\left(\mathrm{m}^{\prime} \approx 4 \mathrm{~m}\right)$
} 
Pour déterminer les éléments du visage, il a déterminé la position des trois points de contact $\mathbf{X}, \mathbf{Z}$ et $\mathbf{N}$ (figure 17), qui sont tous les trois situés sur la forme première.

-la naissance du front $\mathbf{X}$,

-la pointe $\mathbf{Z}$ du nez, intersection de la forme première et de l'arc de cercle de centre $\mathbf{X}$, issu du centre $\mathbf{O}$ et de rayon $\mathbf{2} \mathbf{m}$ '.

-l'extrémité du menton $\mathbf{N}$, intersection de la forme première et de la diagonale issue de $\mathbf{O}$, tracée sur le maillage.

Ainsi, bien que les deux visages soient d'aspect différent, les trois points d'appui, situés sur la forme première, occupent respectivement une place identique dans les deux profils (figure 17). Tout se passe comme si le processus de formation du profil, partant d'un canon presqu'universel, était personnalisé par la diversification.

\section{Diversification}

Cette diversification se manifeste ici, par la commissure des lèvres, par la direction du regard, mais aussi par la forme du nez. Léonard, conscient de cette diversification, énumère, dans son traité de peinture, les différentes sortes de nez. «Il faut, pour cela, se bien souvenir des quatre parties principales du visage, qui sont le menton, la bouche, le front et le nez; et premièrement à l'égard du nez, il s'en trouve de trois différentes sortes; de droits, de concaves ou d'enfoncés, et de convexes ou de relevés. De ceux qui sont droits, il y en a de quatre différentes formes; savoir de longs, de courts, de relevés par le bout, de rabattus; les nez concaves ou camus sont de trois sortes, dont les uns ont leur concavité ou leur enfoncement au haut, d'autres au milieu, et quelques-uns tout au bas; les nez convexes ou aquilins, sont encore de trois sortes, les uns sont relevés vers le haut, quelques autres au milieu, et d'autres en bas; enfin ceux dont la partie relevée est au milieu, peuvent l'avoir droite, ou convexe, ou plate. ${ }^{23}$ "

Ce processus de formation du profil de la tête d'un homme, qui se réalise en trois étapes : forme première, géométrie interne et diversification, peut-il être généralisé aux profils féminins ?

\section{Le portrait d'Isabelle d'Este}

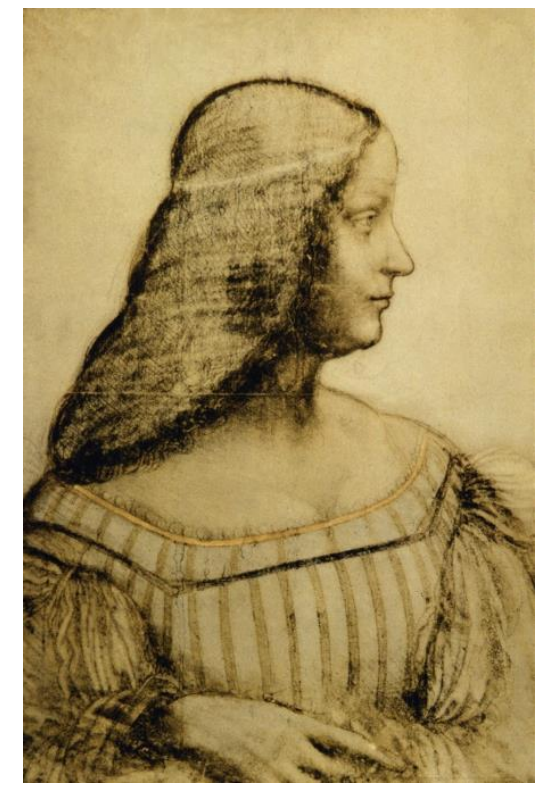

Figure 18. Le portrait d'Isabelle d'Este (Musée du Louvre).

\footnotetext{
${ }^{23}$ Léonard de Vinci[10], chapitre CLXXXIX
} 
Entre décembre 1499 et début mars 1500, Léonard de Vinci a réalisé le portrait d'Isabelle d'Este (Figure 18). C'est un carton conservé au département des arts graphiques du musée du Louvre. Le dessin, de dimensions $63 \times 46,5 \mathrm{~cm}$, en mauvais état de conservation, est réalisé au fusain, à la sanguine, à l'estompe et à la craie ocre, avec des rehauts de blanc.

Isabelle d'Este, marquise de Mantoue, est représentée grandeur nature, les mains croisées, le buste légèrement tourné vers la droite, la tête vue de profil. Ayant eu connaissance du portrait de Cecilia Gallerani,maîtresse de Ludovic le more, effectué par Léonard,elle aurait insisté durant plusieurs années auprès du maître pour qu'il réalise un portrait à son effigie.

\section{La forme première}

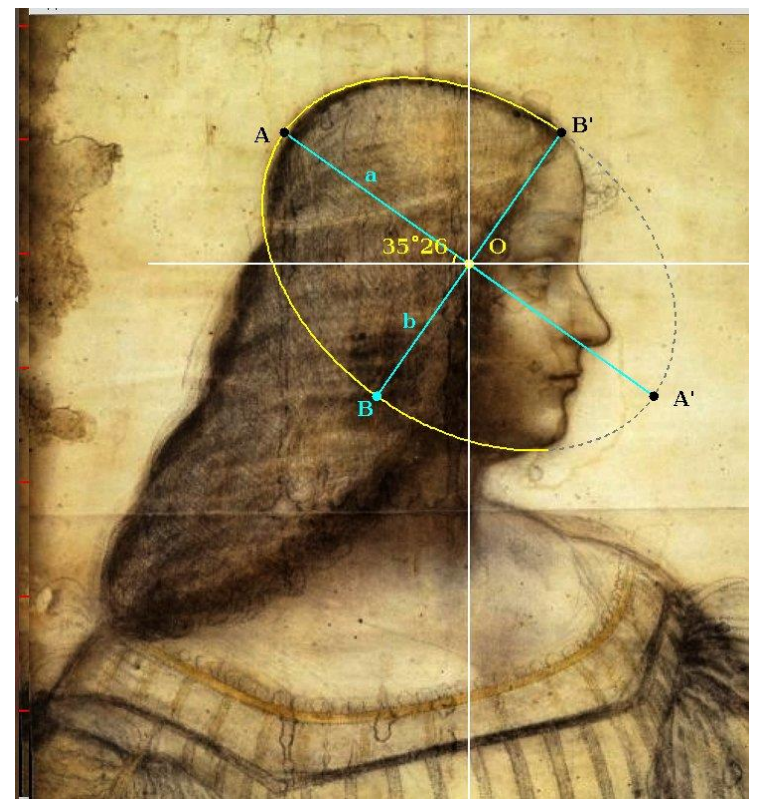

Figure 19. La forme première.

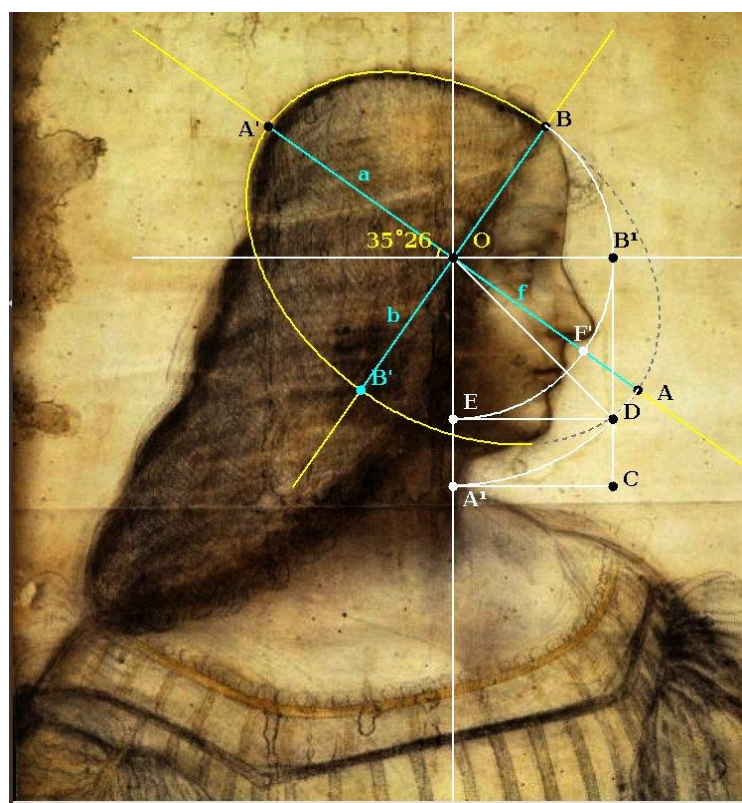

Figure 20. Les paramètres de l'ellipse.

Le contour de la tête, partant du front, passant par le haut de la tête, et entourant le crâne, présente (figure 19) une forme elliptique : c'est la forme première. L'ellipse passe ensuite sous le menton. Le centre $\mathbf{O}$ de cette ellipse se trouve à la hauteur de la pupille de son œil droit. Elle a pour demi-grand axe $\mathbf{a}=\mathbf{O A}$, et pour demi-petit axe $\mathbf{b}=\mathbf{O B}$.

Le grand axe de cette ellipse est incliné d'un angle $\boldsymbol{\beta}=35^{\circ} 26$ par rapport à 1'axe horizontal. Cet angle $\boldsymbol{\beta}$ dont la tangente vaut $\sqrt{\mathbf{2} / \mathbf{2}}$ est caractéristique du maillage harmonique.

En effectuant une rotation des axes de l'ellipse (figure 20), le point $\mathbf{A}$ vient en $\mathbf{A}^{\mathbf{1}}$, le point $\mathbf{B}$ vient en $\mathbf{B}^{\mathbf{1}}$. Le rectangle $\mathbf{O} \mathbf{B}^{\mathbf{1}} \mathbf{C A}^{\mathbf{1}}$ est un rectangle harmonique, car $\mathbf{O A}^{\mathbf{1}}$ est égale à la diagonale $\mathbf{O D} \mathrm{du}$ carré $\mathbf{O B}{ }^{\mathbf{1}} \mathbf{D E}$. La relation entre les paramètres de l'ellipse est telle que : $\mathbf{a}^{\mathbf{2}}=\mathbf{b}^{\mathbf{2}}+\mathbf{f}^{\mathbf{2}}=\mathbf{2} \mathbf{b}^{\mathbf{2}}$. Par suite $\mathbf{f}$ $=\mathbf{b}$, c'est une ellipse particulière. Son excentricité est $\boldsymbol{\varepsilon}=\mathbf{f} / \mathbf{a}=\mathbf{1} / \sqrt{ } \mathbf{2}$. C'est, suivant notre classification, une ellipse de type $\mathbf{1 .}$

La subdivision $(2 \times 2)$ du rectangle harmonique $\mathbf{O B}^{1} \mathbf{C A}^{1}$ détermine un petit maillage harmonique que l'on peut étendre (figure 21) à toute l'image. La maille24 a pour largeur $\mathbf{m}_{\mathbf{h}}$ et pour hauteur $\mathbf{m}_{\mathbf{v}}$. Le demi-grand axe a est égal à la hauteur de deux mailles : $\mathbf{a}=\mathbf{2 m}_{\mathbf{v}}$. Le demi-petit axe $\mathbf{b}$ et la distance focale $\mathbf{f}$ sont égaux à la largeur de deux mailles : $\mathbf{b}=\mathbf{f}=\mathbf{2 m}_{\mathbf{h}}$.

Sur ce maillage, le centre de la pupille de son œil droit coïncide précisément avec un nœud du maillage et les lèvres sont situées une hauteur de maille en-dessous.

\footnotetext{
${ }^{24}$ En se référant aux dimensions du carton, nous estimons $\mathrm{mh}=4,59 \mathrm{~cm}$ et $\mathrm{mv}=6,55 \mathrm{~cm}$ 
Dans cette ellipse de type 1 , qui possède des propriétés particulières ${ }^{25}$, on peut inscrire un cercle passant par les foyers (figure 21). Le cercle part du haut du front au point B, passe par l'extrémité du nez et le bout des lèvres.

Ensuite, Léonard, s'appuyant sur la forme première et la géométrie interne, a pu, en appliquant toute sa virtuosité, dessiner le pourtour du visage, et même donner au personnage sa ressemblance.

\section{La Belle Princesse (1496)}

Quatre ans plus tôt, Léonard a réalisé à la demande de Ludovic Sforza, un dessin ${ }^{26}$ représentant sa fille Bianca Sforza (1482-1497), dite La Belle Princesse. Le dessin a été effectué sur vélin de dimensions (33 x $24 \mathrm{~cm}$ ), au moyen de trois crayons (la pierre noire, la craie blanche et la sanguine), et aussi avec de l'encre.

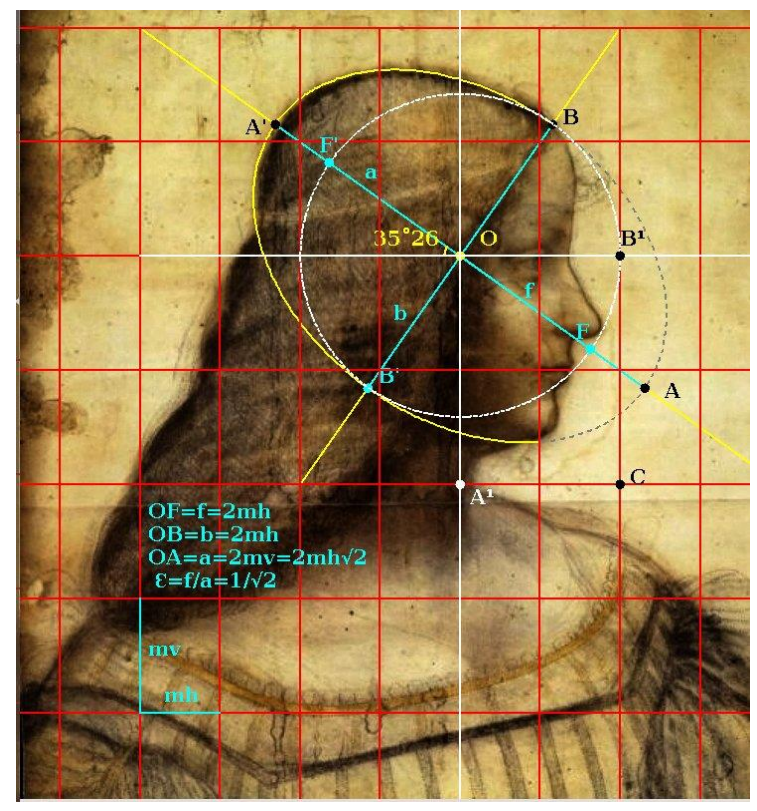

Figure 21. Le maillage harmonique et la valeur des paramètres de l'ellipse

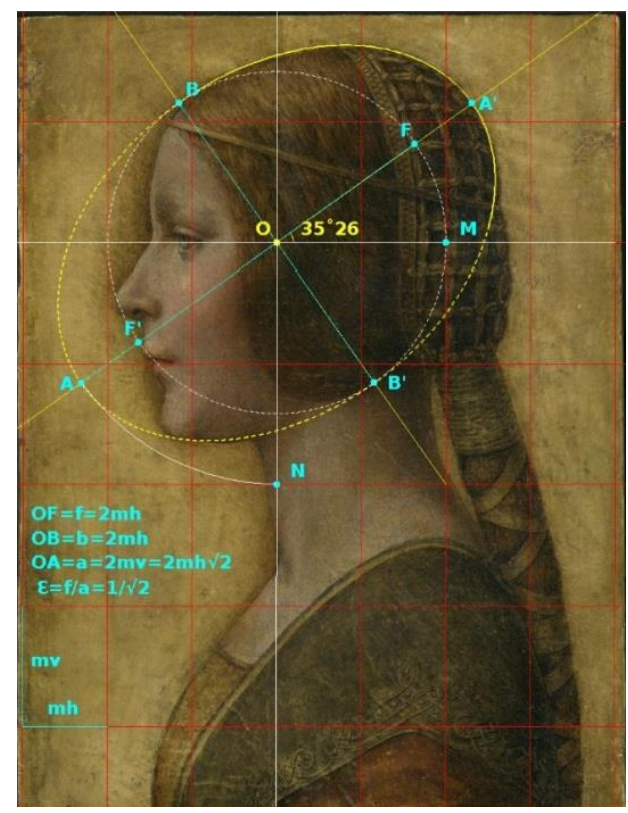

Figure 22. La tête de la Belle Princesse possède la même forme première que celle d'Isabelle d'Este

Bianca Sforza est représentée de profil. Son regard fixe est dirigé vers la gauche. Elle ne sourit pas. Cette œuvre, fait partie des cinq portraits féminins que nous avions analysés dans une étude précédente $^{27}$. Le haut de la chevelure satin claire de la jeune fille (14 ans) présente une forme elliptique qui continue, en passant par le dessous du menton (figure .22). Cette forme elliptique tracée sur un maillage harmonique, correspond à une forme première. Elle présente la même excentricité que la forme première du profil d' Isabelle d'Este. Comme cette dernière, elle est inclinée d'un angle de $35^{\circ} 26$. Les deux formes premières sont semblables et disposées de façon symétrique. Cependant les dimensions ${ }^{28}$ de La Belle Princesse sont deux fois plus petites.

Léonard n'a donc pas pu utiliser le même poncif. Mais là encore, le centre de la pupille de son œil droit coïncide précisément avec le même nœud du maillage et les lèvres sont situées une hauteur de maille en-dessous. De la même façon, le cercle inscrit partant du front au point $\mathbf{B}$, passe par l'extrémité du nez et le bout des lèvres.

\footnotetext{
${ }^{25}$ Crettez J-P. , [6]

${ }^{26}$ Le dessin est actuellement conservé à la bibliothèque Narodowa à Varsovie.

${ }^{27}$ Crettez J-P.,[4], \$ 8.2.3

${ }^{28}$ En se référant aux dimensions physiques du vélin nous pouvons estimer $m_{h}=2,65 \mathrm{~cm}$ et $m_{v}=3,75 \mathrm{~cm}$
} 
Ainsi, bien que les deux personnages soient différents par leur âge, leur état et leur condition, les deux profils de femme ont été construits à partir de la même forme première selon la même démarche. Bien plus, c'est la même démarche que celle utilisée pour dessiner les profils masculins.

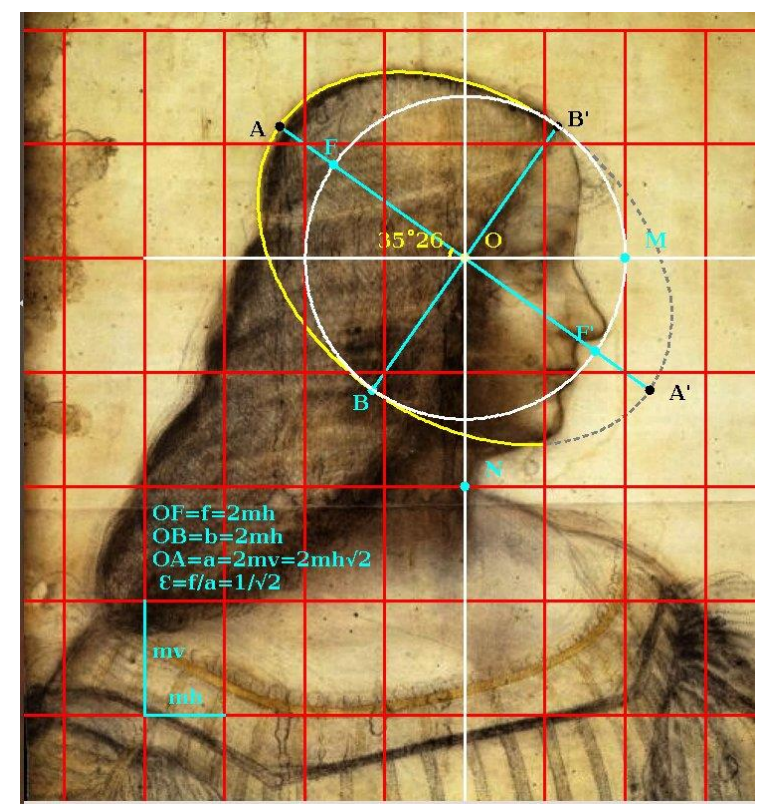

Figure 23. Le portrait d'Isabelle d'Este et sa forme première

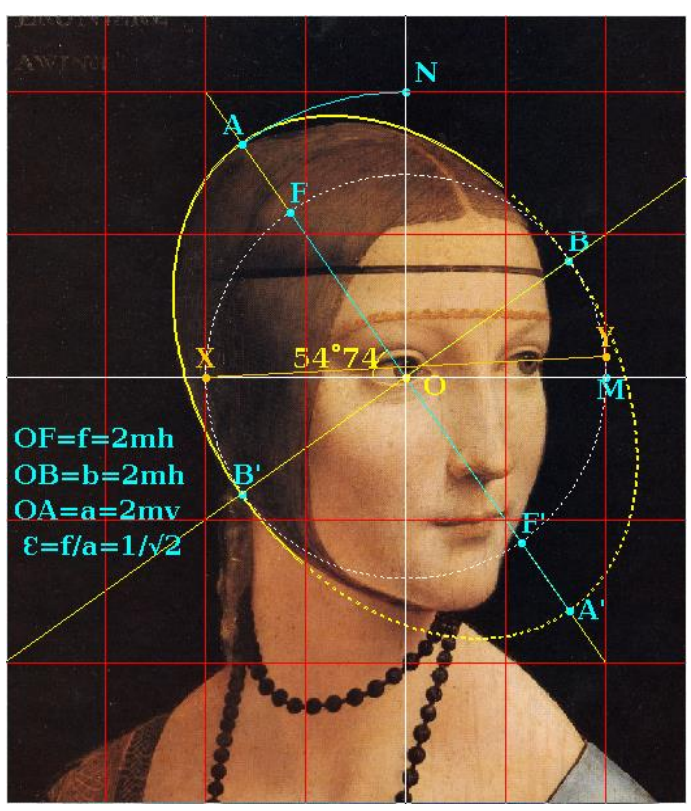

Figure 24. La tête de la Dame à l'hermine et sa forme première.

\section{Extension de la démarche aux portraits}

La Dame à l'hermine (1488-1490) est un tableau considéré comme le portrait de Cecilia Gallerani (1473-1536). Il fait partie des cinq portraits que nous avions analysés dans cette étude précédente. Ce n'est pas le dessin d'un profil, mais un portrait peint, vu de trois quarts. Néanmoins, nous avons montré que le contour du crâne présente (Fig. 23) une forme elliptique que l'on peut associer à une forme première. Cette dernière possède la même excentricité $\boldsymbol{\varepsilon}=\mathbf{1} / \sqrt{\mathbf{2}}$ que la forme première du profil d'Isabelle d'Este. Son grand axe est incliné d'un angle $\boldsymbol{\alpha}=\mathbf{5 4} \mathbf{7 4}^{\circ}$ par rapport à l'axe horizontal. Il est perpendiculaire au grand axe de la forme première de La Belle Princesse.

Nous avons également montré pour chacun des trois autres portraits que nous avons analysés, le contour supérieur de la tête de chacun de ces cinq personnages correspondait de façon précise à un arc d'ellipse qui appartenait comme ci-dessus à une forme première. Ces formes premières ont des excentricités différentes. Par exemple, le portrait de Ginevra de' Benci possède une forme première dont l'excentricité est égale à 0,6. Il a été peint vers (1474-1476), bien avant sa rencontre avec Pacioli.

\section{Forme première composée}

Ainsi, la démarche de Léonard semble être restée la même pendant toute sa carrière de peintre, depuis le portrait de Ginevra de' Benci jusqu' au portrait de la Joconde. Cependant dans les dernières années de sa carrière artistique, Léonard réalise ses portrait avec une forme première composée, constituée de deux formes elliptiques. Mais pour respecter l'harmonie des proportions, ces deux formes elliptiques doivent être semblables, c'est-à-dire avoir même excentricité. Dans le portrait de la Joconde, la forme elliptique de la tête et celle du voile de gaze ont pour excentricité $\boldsymbol{\varepsilon}=\mathbf{1 / 2}$. La deuxième est $\sqrt{2}$ fois plus petite que la première, et sa surface vaut la moitié. Les deux formes elliptiques, sont consonantes entre elles. Elles résonnent à l'octave. 
C'est aussi le cas du Salvator Mundi ${ }^{29}$, où la forme première composée est constituée de la forme elliptique de la tête et de celle du visage. Elles ont pour excentricité $\boldsymbol{\varepsilon}=\mathbf{1} / \sqrt{ } \mathbf{2}$. Là encore, les deux formes elliptiques, sont consonantes entre elles. Elles résonnent à l'octave.

\section{Conclusion}

L'analyse de ce simple dessin dépourvu d'explications, nous a permis d'entrevoir la démarche géométrique suivie par Léonard pour réaliser les 4 profils que nous avons analysés. Le maître, à la recherche des mécanismes de création des formes procédait en trois étapes. Il commençait par tracer sur un maillage carré ou harmonique, une ellipse constituant la forme première, qui initialisait une partie du profil cherché, lui donnait sa forme globale, mais aussi, sa position, sa taille et son orientation. Puis, à partir des paramètres de cette ellipse et du support sur laquelle elle a été tracée, il établissait avec précision la géométrie interne qui permettait de préciser d'autres formes spécifiques au profil recherché, et de définir les points d'appui. Enfin, il personnalisait le dessin en adaptant le reste du tracé à la particularité de la tête du personnage, tout en conservant les proportions.

Généralisant cette démarche, nous avons montré qu'il était possible d'assimiler à une forme première chacune des formes elliptiques que nous avons mises en évidence lors de l'analyse des 5 portraits féminins ${ }^{30}$ que nous avions étudiés précédemment, mais également lors de l'analyse du Salvator Mundi. Ainsi, la forme première (représentation géométrique de l'esquisse) serait bien à la base de la démarche constructive de Léonard pour réaliser ses portraits.

Mais si la géométrie permet de simuler les mécanismes qui donnent forme aux produits de la nature, la peinture doit montrer la perfection de ses produits :" La peinture ne se contente donc pas de "refaire" la nature en se fondant sur la connaissance de ses lois, elle "achève" la nature et le "tableau est le seul moyen d'obtenir la collaboration intégrale des formes et d'en dégager l'accord final." 31

\section{Bibliographie}

[1] Arasse D., Léonard de Vinci, Édition Hazan, Paris, 1993.

[2] Bouleau Ch., Charpentes : La géométrie secrète des peintres, Le Seuil, Paris, 1963.

[3] Clark Kenneth., Léonard de Vinci Librairie générale française 1967

[4] Crettez J-P., Les supports de la géométrie interne des peintres : de Cimabue à G. de La Tour. Éditions ISTE (2017).

[5] Crettez J-P., Openscience -Géométrie interne d'une "Nuit" de G. de La Tour : "L'Apparition de l'ange à saint Joseph" (musée des beaux-arts, Nantes), vol 2 -Numéro 1

[6] Crettez J-P., Openscience - Art et science- Géométrie interne du "Salvator Mundi", vol 3 -Numéro 1

[7] Kemp Martin., Leonardo, Oxford University Press, 2004

[8] Mottin, Bruno., Léonard de Vinci et l'art du dessin: une approche de laboratoire. Léonard de Vinci, Éditions Hazan 2019

[9] Léonard de Vinci., Manuscrit A, Bibliothèque de l'Institut de France

[10] Léonard de Vinci., Traité de la peinture. Chez Deterville, rue du Battoir, Paris

[11] Léonard de Vinci, Le traité de peinture, note 354, p. 149. Éditions Jean de Bonnot, Paris 1982

\footnotetext{
${ }^{29}$ Crettez J-P.,[6]

${ }^{30}$ Crettez J-P.,[4], \$ 8.2.3

${ }^{31}$ Arasse.,[1], p. 215
} 